\title{
Some theoretical and computational aspects of nanocluster structure solution
}

\author{
Simon J. L. Billinge \\ Department of Applied Physics and Applied Mathematics, Columbia University, \\ Condensed Matter and Materials Science Department, Brookhaven National Laboratory \\ Phil Duxbury \\ Department of Physics and Astronomy, Michigan State University
}

\begin{abstract}
A revolution in materials science followed the invention of $x$-ray crystallography at the beginning of the 20th century. The importance of knowing the structure of a material cannot be overstated. Yet we still struggle to solve the structures of nanoscale finite objects, though powerful new methods are emerging. We have been thinking about this problem by looking at the mathematical underpinnings of the problem beyond the crystallographic case, where translational symmetry and the resulting space-groups provide a sound theoretical foundation for studying the problem. Starting from a graph-theoretic representation, and treating structures as a point cloud in euclidean space, we can couch the nanostructure inverse problem in terms of a distance geometry problem. The kind of information obtained in a scattering experiment is effectively an unassigned list of distances (the pair distribution function). Solving the NIP then becomes the task is to discover the assignments of nodes and edges on the graph, and then finding an embedding in Euclidean space. This is the socalled unassigned distance geometry problem. In this presentation I will describe this, and explain some algorithms that then present themselves for solving the problem. I will discuss limitations that are becoming apparent but will present some practical solutions and directions that present themselves.
\end{abstract}

